\title{
Percutaneous tricuspid edge-to-edge repair — patient selection, imaging considerations, and the procedural technique. Expert opinion of the Working Group on Echocardiography and Association of Cardiovascular Interventions of the Polish Cardiac Society
}

\author{
Adam Rdzanek', Piotr Szymański², Andrzej Gackowski³, Piotr Scisło', Jerzy Pręgowski, Arkadiusz Pietrasik1, \\ Jarosław Trębacz ${ }^{5}$, Karol Zbroński', Janusz Kochman'1, Adam Witkowski ${ }^{4}$, Wojciech Wojakowski ${ }^{6}$, Marek Grygier ${ }^{7}$ \\ Reviewers: Aleksander Araszkiewicz', Stanisław Bartuś8, Maciej Dąbrowski', Marcin Fijałkowski \\ (on behalf of the Polish Cardiac Society) ${ }^{9}$, Michał Hawranek ${ }^{10}$, Zenon Huczek', Paweł Kralisz ${ }^{11}$, Jacek Kusa ${ }^{12}$, \\ Katarzyna Mizia-Stec ${ }^{13}$, Zofia Oko-Sarnowska ${ }^{14}$, Tomasz Roleder ${ }^{15}$, Grzegorz Smolka ${ }^{6}$ (on behalf of the Polish

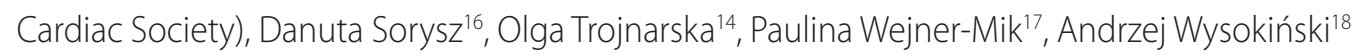

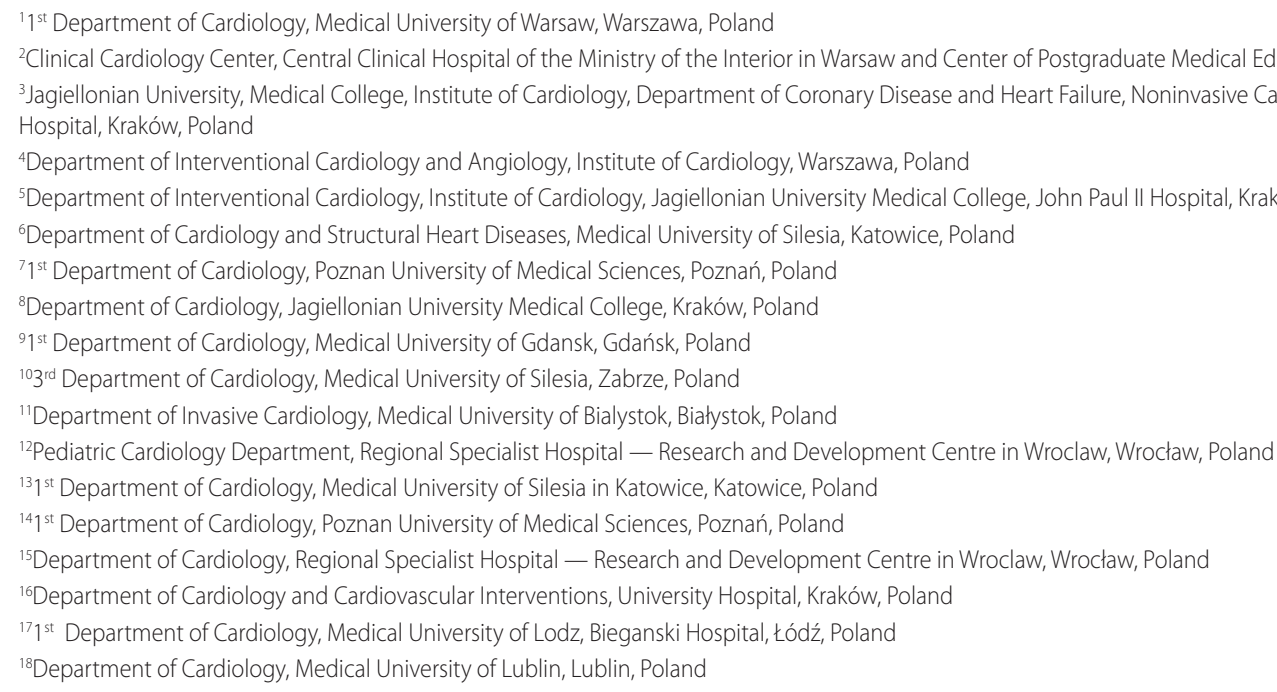

\author{
Correspondence to: \\ Prof. Piotr Szymański, MD, FESC, \\ Clinical Cardiology Center, \\ CSK MSWiA in Warsaw, \\ Wołoska 137, 02-507 \\ Warszawa, Poland, \\ phone: +48 477221830 , \\ e-mail: \\ pszymanski@ptkardio.pl \\ Copyright by the Author(s), \\ 2021 \\ Kardiol Pol. 2021; \\ 79 (10): 1178-1191 \\ DOI: 10.33963/KP.a2021.0125 \\ Received: \\ September 18, 2021 \\ Revision accepted: October \\ 4, 2021 \\ Published online: \\ October 5, 2021
}

\section{A B S T R A C T}

Tricuspid regurgitation (TR) is a common acquired valvular heart disease (VHD). TR has a progressive character and is associated with impaired long-term survival in both symptomatic and asymptomatic subjects. Despite this knowledge, the overall number of tricuspid valve surgeries is very low worldwide, and many patients with clear indications for intervention are left untreated. The development of less invasive transcatheter techniques may offer new treatment options in this growing population of patients. Out of various percutaneous methods proposed, tricuspid edge-to-edge repair has recently gained considerable attention. This article summarizes available data regarding this new treatment method.

Kardiol Pol 2021; 79, 10: 1178-1191 


\section{INTRODUCTION}

Tricuspid regurgitation (TR) is a common acquired valvular heart disease (VHD). Its prevalence is rapidly growing with the aging population, as a recent population study indicates its prevalence nearly equals that of severe aortic stenosis [1]. In most cases, TR has a functional character frequently secondary to left-sided congestive heart failure or VHD and a subsequent right ventricular enlargement. In a growing number of patients with preserved right ventricular function, an alternative mechanism of TR due to tricuspid annulus dilatation in the setting of longstanding atrial fibrillation has been reported [2]. Finally, secondary functional TR develops in a large portion of patients previously operated on for mitral or aortic valve disease, causing the recurrence of heart failure symptoms [3].

Tricuspid regurgitation is a progressive disease that is associated with impaired long-term survival in both symptomatic and asymptomatic subjects $[4,5]$. The presence of severe TR is associated with a nearly 2 -fold increase in the mortality rate of heart-failure patients. The reported overall 10-year survival rate is lower than 40\% [6]. Moreover, a linear correlation between regurgitation severity, defined as effective regurgitant orifice area, and mortality has recently been reported [7].

The presence of a moderate or worse TR is a well-established risk factor and affects the long-term survival in patients undergoing both surgical and percutaneous procedures for left-sided VHD [8]. TR is not only a marker of disease severity but also a potential target for therapeutic intervention and thus current guidelines advocate using a liberal approach for a concomitant tricuspid valve (TV) repair in patients undergoing surgical treatment of left-sided VHD.

Despite this knowledge, the overall number of tricuspid valve surgeries is very low worldwide, and many patients with clear indications for intervention are left untreated. The development of less invasive transcatheter techniques may offer new treatment options in this growing population of patients. Out of various percutaneous methods proposed, tricuspid edge-to-edge repair has recently gained considerable attention.

\section{EDGE-TO-EDGE REPAIR FOR TRICUSPID REGURGITATION}

During the last decade, percutaneous edge-to-edge repair has become a well-established treatment method in patients with mitral valve insufficiency. In approximately $25 \%-30 \%$ of patients referred for this therapy, concomitant severe TR is also documented. The treatment of mitral regurgitation alone led to a reduction of TR grade in only $30 \%-40 \%$ of cases [9], therefore the development of edge-to-edge tricuspid repair was a natural next step in the therapy of patients with concomitant mitral and tricuspid disease.

The first percutaneous tricuspid edge-to-edge repair was performed under the off-label use of the MitraClip sys- tem (Abbott Vascular, Santa Clara, CA, USA) via the jugular vein in 2015 , followed shortly by successful transfemoral procedures [10]. An early feasibility study that was conducted in 64 patients with both isolated TR or concomitant mitral and tricuspid insufficiency and deemed unsuitable for surgery, showed high periprocedural success with $97 \%$ tricuspid clip implantation rate and $91 \%$ patients having at least one grade reduction in TR severity. The therapy proved to be safe with no periprocedural complications and effective in terms of the New York Heart Association (NYHA) class and exercise tolerance improvements [11].

Further studies have indicated a durable reduction to a moderate or less TR at 12-month follow-up. This was observed in $72 \%$ of patients and accompanied by a $22 \%$ reduction in heart failure hospitalizations and an improvement of a 1-year survival from $60 \%$ to $79 \%$ when compared to patients who failed a TR repair attempt [12]. Furthermore, in the absence of randomized data, a recent retrospective cohort study showed that the tricuspid edgeto-edge repair with the MitraClip NT system combined with the optimal medical therapy in patients with severe TR significantly reduced mortality ( $24.9 \%$ vs. $53.1 \%$; median 14-month follow-up) when compared to the medical therapy only [13].

\section{POSSIBLE CLINICAL INDICATIONS FOR PERCUTANEOUS EDGE-TO-EDGE THERAPY FOR TRICUSPID REGURGITATION}

The recently published guidelines of the European Society of Cardiology concerning the management of valvular heart disease introduced for the first time the possibility of percutaneous intervention in patients with TR. According to the document, the intervention may be considered by the local Heart Team at experienced centers in symptomatic, inoperable, and anatomically eligible patients. However, because of the data paucity, no specific recommendations regarding different clinical situations are given [14].

In patients with TR, we usually deal with two clinical scenarios: coexistence of tricuspid and mitral regurgitation in a patient scheduled for edge-to-edge mitral valve repair or a patient with isolated TR. Due to the lack of exact indications concerning percutaneous treatment, guidelines on surgical treatment might prove to be helpful in everyday clinical decision-making.

In the first scenario, according to the current guidelines, patients scheduled for left-sided valve surgery should undergo concurrent tricuspid repair if a severe TR is present.

This strategy should also be considered in subjects without significant TR but with a dilated tricuspid annulus (i.e., $\geq 40 \mathrm{~mm}$ or $>21 \mathrm{~mm} / \mathrm{m}^{2}$ by $2 \mathrm{D}$ echocardiography), or with current or previous symptoms and signs of right-sided heart failure, as in such cases, the probability of TR progression during follow-up is substantial. This approach does not add risk to the index surgical intervention while reducing the need for higher risk re-intervention [15]. As previously said, up to $30 \%$ of patients treated with MitraClip for mitral 
regurgitation (MR) have significant TR. The presence of TR independently worsens prognosis after isolated mitral edge-to-edge repair. There is only a moderate probability that successful MR repair will improve TR. However, it is possible to correct TR simultaneously by the off-label use of the same MitraClip device provided that the tricuspid valve anatomy is suitable, and echo visualization is of good quality. The results of several registries suggest that this strategy reduces heart failure symptoms and the total dose of diuretics. Recently the pooled data of patients with significant MR and TR from TRAMI and TriValve registries were retrospectively analyzed. Subjects enrolled in TriValve underwent concurrent mitral and tricuspid treatment while patients observed in the TRAMI registry only had an isolated mitral intervention performed. At one-year follow-up, concurrent TR treatment was independently associated with lower mortality (16.4\% vs. $34 \% ; P=0.035)[16,17]$. Nevertheless, further controlled randomized studies are needed to confirm these promising observations.

According to available data, in our opinion, concurrent edge-to-edge correction of TR in patients scheduled for MR treatment may be considered in patients with good echocardiographic visualization of the tricuspid valve, favorable valve anatomy, and:

- moderate, if at least two of the specific criteria of severe TR are met (or borderline values are observed) and valve is significantly remodelled ("moderate-to-severe" $\mathrm{TR}$ ), severe or greater TR regardless of symptoms of right-sided heart failure;

- moderateTR in patients with current or previous symptoms or signs of right-sided heart failure.

Currently, we do not recommend edge-to-edge tricuspid repair in patients with less than moderate TR and annular dilatation. These patients should be closely observed and if the TR progresses possibly scheduled for a staged isolated TR correction with a dedicated device.

In the case of patients with isolated TR, open-heart surgery is associated with a high periprocedural mortality risk that reaches $10 \%-25 \%$ in severely symptomatic patients [18]. This may be the result of unfavorable baseline clinical characteristics of the patients, who often suffer from secondary liver and/or renal failure. According to the current guidelines, surgery should be considered in patients with isolated significant TR with signs and symptoms of right-sided heart failure. The procedure should be optimally performed before the onset of right ventricular dysfunction or end-organ failure. The surgery might also be considered in patients with prior left-sided valve surgery and isolated TR but without right ventricular dysfunction or severe pulmonary hypertension. However, given the high surgical risk, many symptomatic patients with severe TR are denied surgery. Percutaneous TR treatment with an edge-to-edge technique may be a valid option for this population.

In our opinion, in the case of isolated TR, percutaneous edge-to-edge repair might be considered by the Heart Team in symptomatic, high surgical risk patients with mod- erate to severe TR, favorable valve anatomy, good echocardiographic valve visualization, and without significant pulmonary hypertension. The expected life expectancy should exceed 12 months to avoid futility.

\section{ECHOCARDIOGRAPHIC ASSESSMENT OF THE TRICUSPID VALVE IN THE CONTEXT OF INTERVENTION}

When compared to other valves, the tricuspid valve has complex and variable anatomy. It is usually composed of three separate leaflets: an anterior and septal leaflet in addition to a smaller posterior leaflet; however, accessory leaflets can be also present. Only the septal portion of the annulus is relatively solid, while the remaining part is prone to dilatation, which is a typical reason for functional TR. The regurgitant orifice can be central, but in most cases, it has a complex shape due to pericommissural leakage. Primary and secondary chords connected to small and variable papillary muscles or directly to the myocardium can cause leaflet restriction - another mechanism of TR.

Both the thin leaflets and the subvalvular apparatus are poorly echogenic. The valve is located off axis from the esophagus (Figure 1). That is why, when compared to the mitral, the tricuspid valve is a much more demanding imaging target. Although multiple transthoracic (TTE) and transesophageal (TEE) 2D views, accompanied by biplane and 3D imaging, can overcome this problem, obtaining sufficient imaging quality for approx. $20 \%$ of patients may not be possible. Even for experienced echocardiographers, specific training is needed to distinguish the three leaflets and assess the valve's repairability. It is even more demanding to select the implantation target, provide navigation images, and check the coaxiality/perpendicularity of the clip prior to implantation. Ventricular pacing leads are frequently present in TR patients and require special attention due to their influence on the mobility of the leaflets. The presence of the pacemaker leads, especially when colliding with the valve leaflets, decreases the chances of a successful procedure. Commissural rather than central position can be accepted in patients referred for edge-to-edge repair.

A detailed TTE evaluation requires TV parasternal long- and short-axis views, an apical 4-chamber view with anterior and posterior modifications, and substernal views to distinguish the TV leaflets (Figure 2). The mechanism and localization of the main TR orifice, as well as TR severity, should be assessed (Table 1). In candidates for edge-toedge intervention, TEE is needed to make sure the valve is sufficiently visible (when the patient is positioned on their back) and to pre-plan the valve clipping prior to the qualification for the procedure.

Understanding the orientation of the specific views and meaning of the anatomical landmarks are helpful both in TTE and TEE in distinguishing the leaflets and to localize the commissures (Figure 2). It can be practiced using a simulator - the most important images are explained in Figures 3-6. The antero-septal commissure is located close to the 


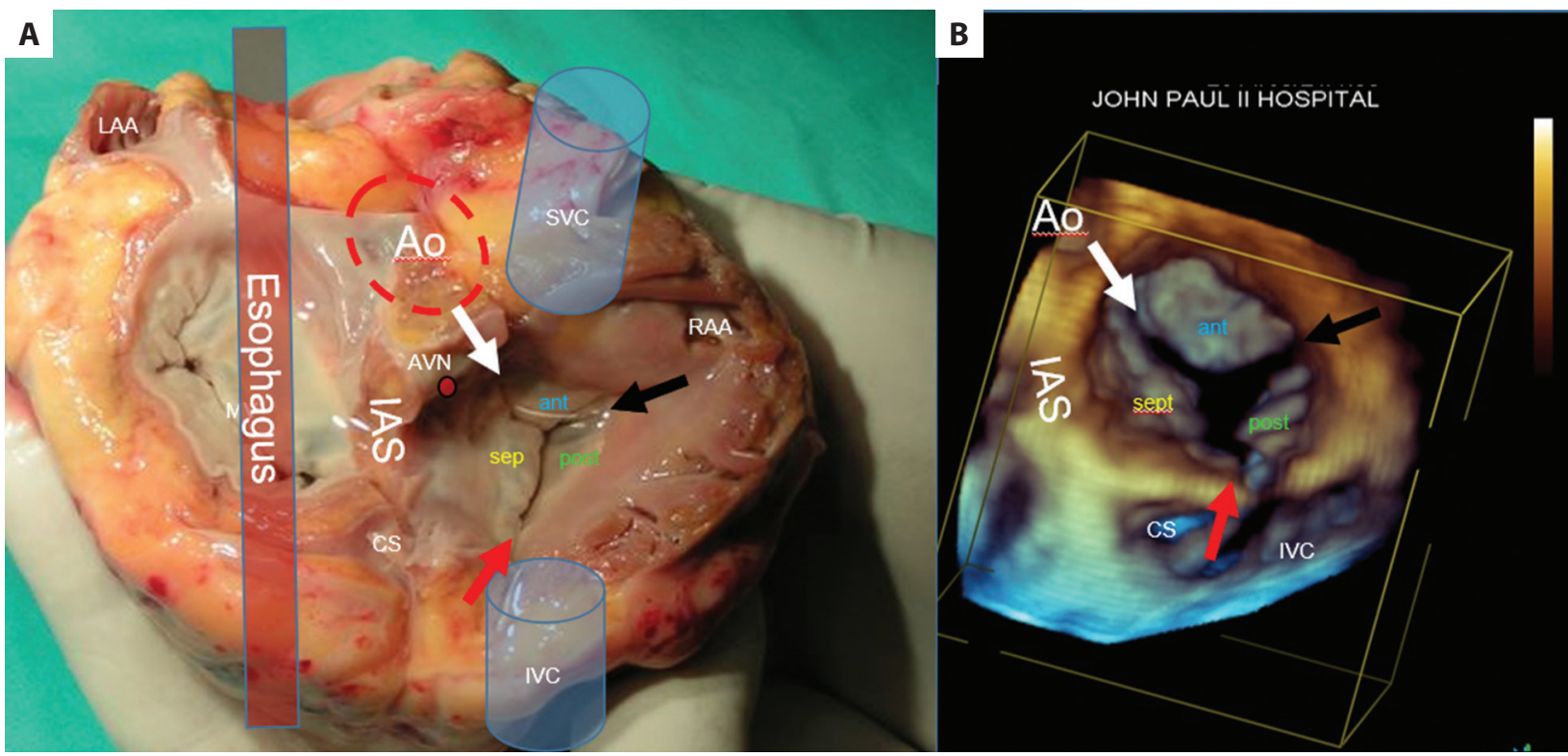

Figure 1. Anatomical specimen of the heart without atrial walls (A). Esophagus is located above the mitral valve, and the TV has to be imaged in an oblique way. Corresponding 3D view of the TV. The commissures are marked with arrows. Note anatomical landmarks: Ao, IAS, CS, IVC. Position of the AVN is also shown (B)

Abbreviations: ant, anterior; Ao, aorta; AVN, atrioventricular node; CS, coronary sinus; IAS, interatrial septum; IVC, inferior vena cava; LAA, left atrial appendage; post, posterior; RAA, right atrial appendage; TV, tricuspid valve; sept, septal; SVC, superior vena cava
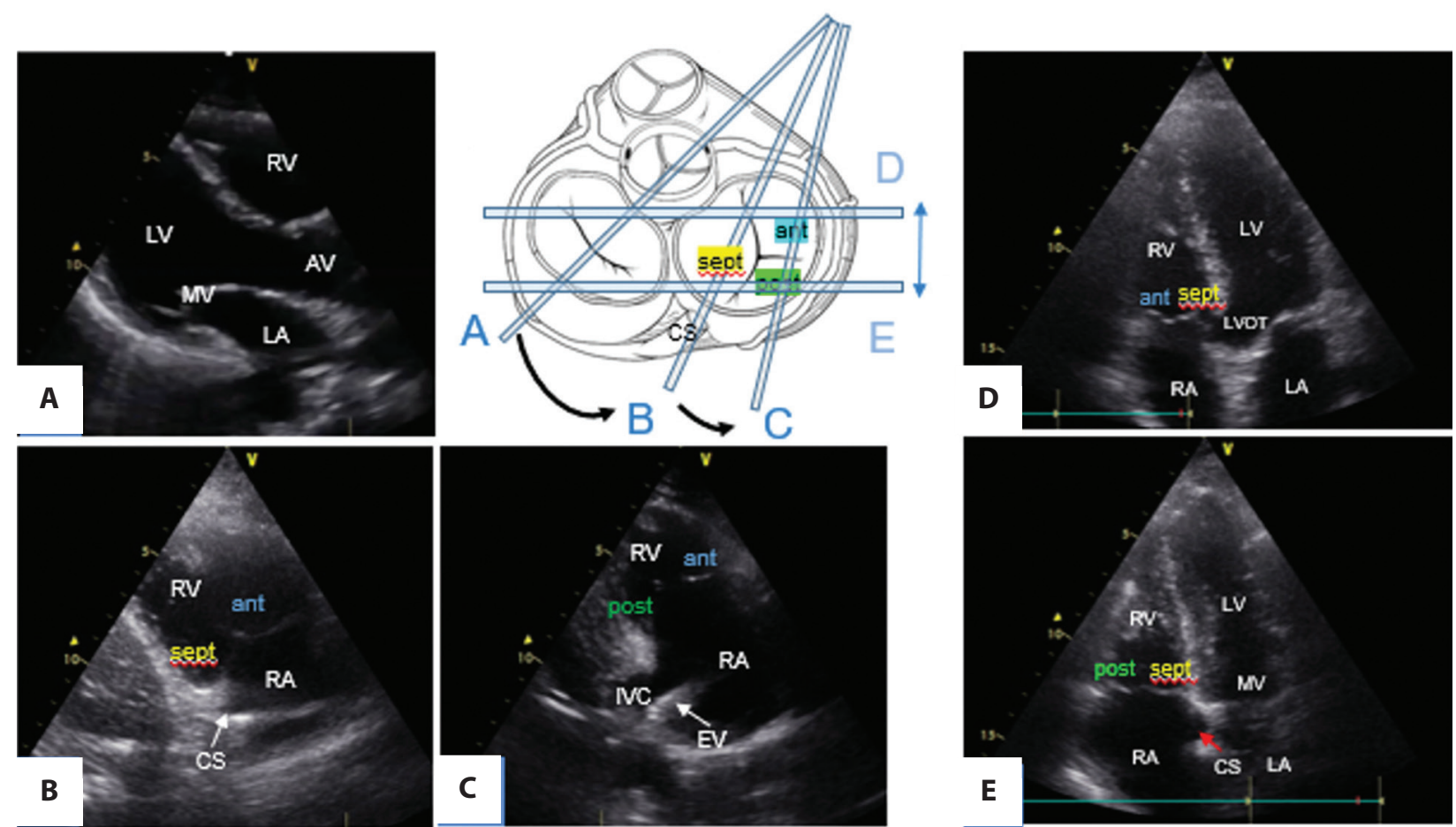

Figure 2. Main TTE views modified to differentiate the tricuspid valve leaflets. Left panel: Parasternal long axis view (A) modified to visualize the anterior and septal (B) or anterior and posterior leaflets (C). Right panel: 4-chamber view tilted anteriorly (D) and posteriorly (E). Note the landmarks - CS, IVC with EV and LVOT

Abbreviations: EV, Eustachian valve; LVOT, left ventricle outflow tract; TTE, transthoracic echocardiography; other — see Figure 1

aortic valve (next to the N-R commissure), and the postero-septal commissure lies between the coronary sinus and inferior vena cava (Figure 1). Due to anatomical limitations, 2D images cannot be fully coaxial with the trajectory of the device. If the imaging quality is sufficient, it can be solved by multiplanar live 3D imaging.

\section{ECHOCARDIOGRAPHIC CRITERIA OF PATIENT SELECTION}

Two systems are currently approved (CE mark) for edgeto-edge tricuspid valve repair in Europe - TriClip and Pascal. According to the Instructions for Use, the TriClip 
Table 1. Echocardiographic criteria of TR severity table modified, based on [19-21]. The most important parameters are marked in bold

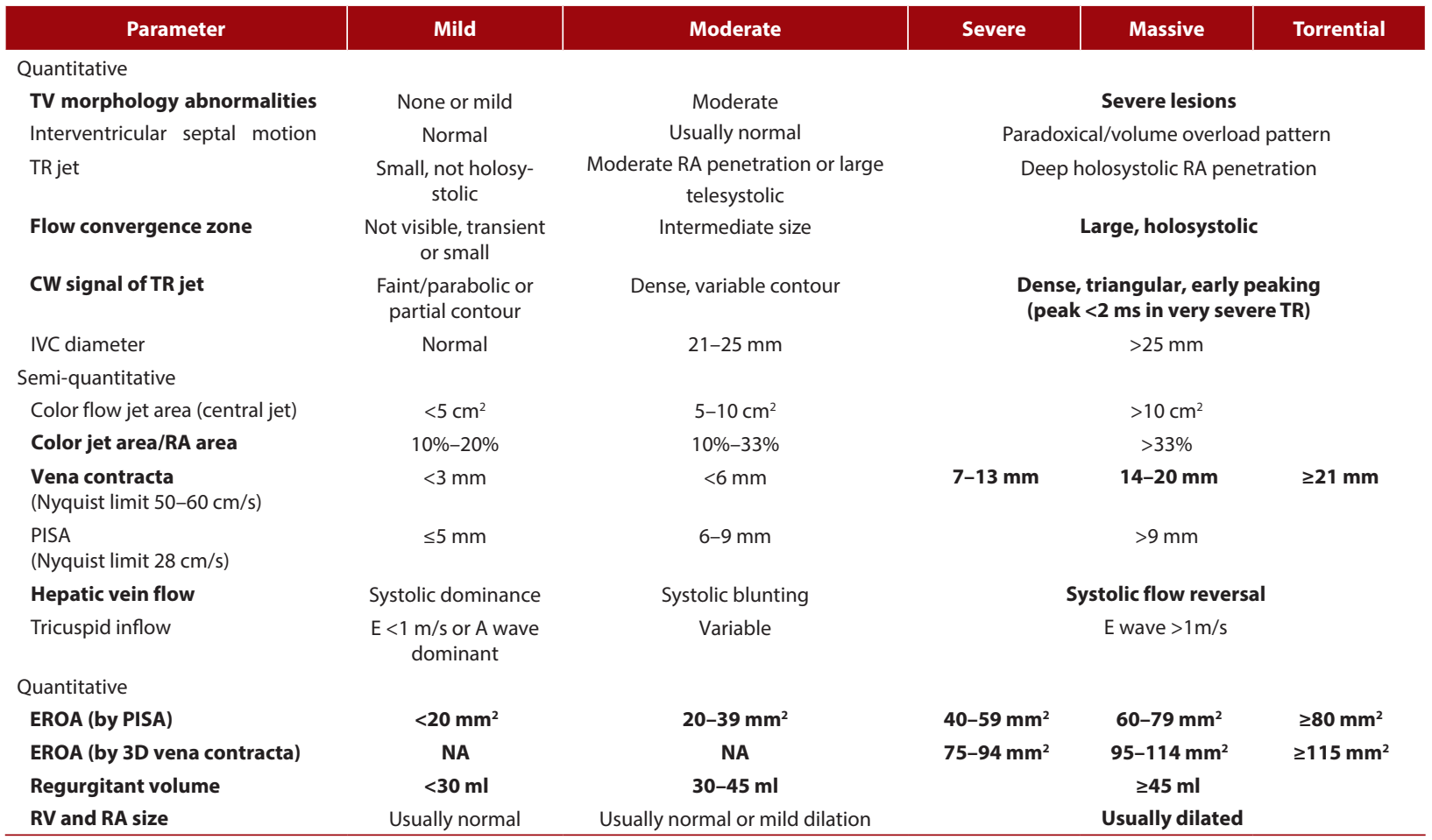

Abbreviations: CW, continuous wave; EROA, effective regurgitant orifice area; IVC, inferior vena cava; NA, not applicable; PISA, proximal isovelocity surface area; RA, right atrium; RV, right ventricular; TR, tricuspid regurgitation; TV, tricuspid valve
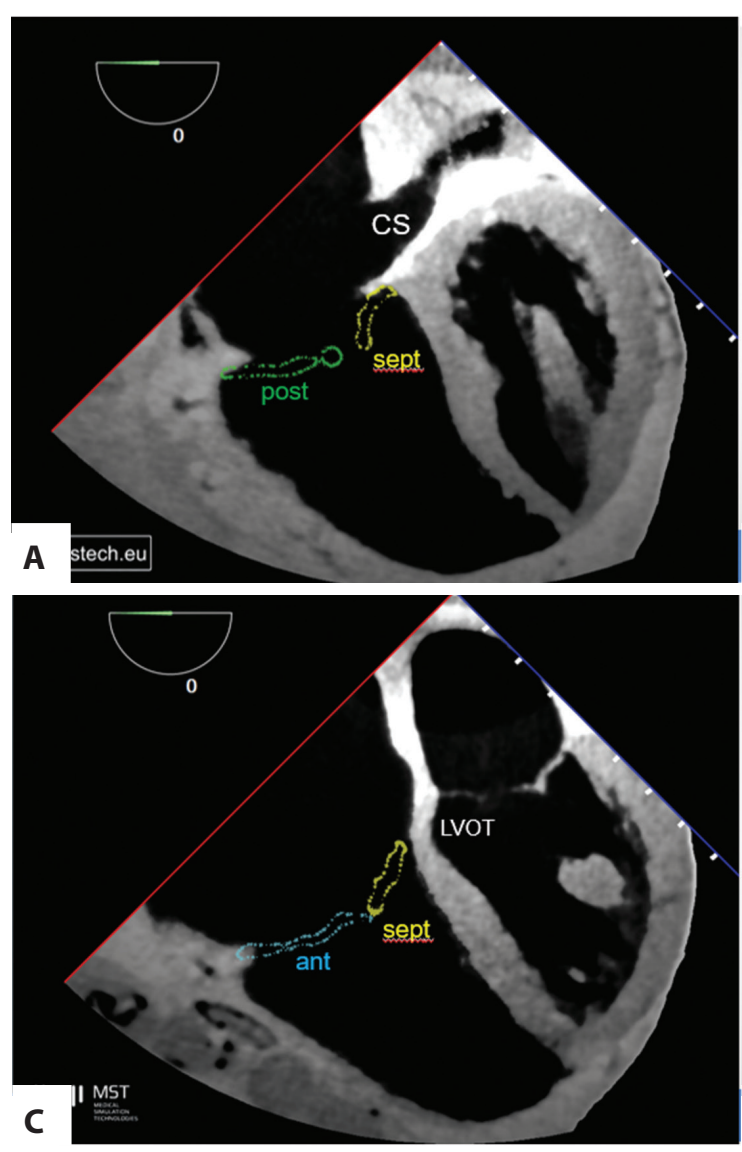

B
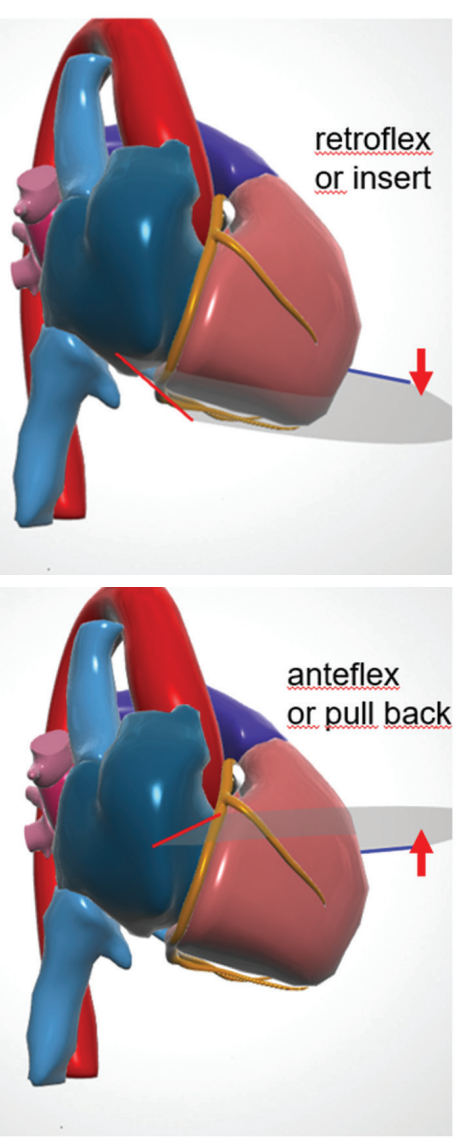

Figure 3. Simulation of the mid-esophageal $0^{\circ} 4$-chamber modified by retroflexing $(\mathbf{A})$ or anteflexing the probe $(\mathbf{B})$ to differentiate the posterior from the anterior leaflet, both coapting with the septal leaflet. Similar views can be obtained by inserting or pulling back the probe or by tilting the TTE 4-chamber plane (Figure 2D and 2E). MrTEEmothy Simulator was used (Medical Simulation Technologies)

Abbreviations: see Figures 1 and 2 

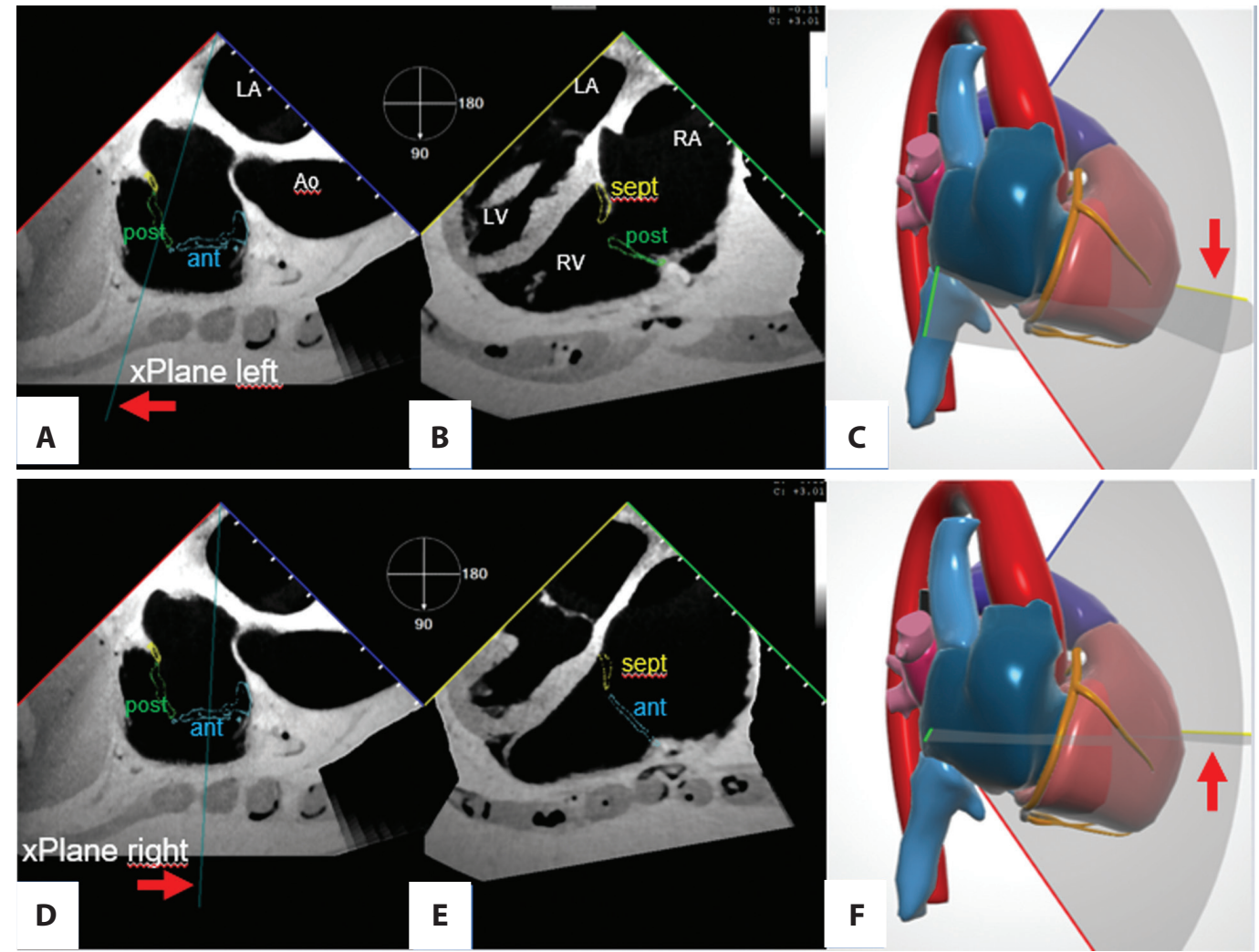

Figure 4. Simulation of the mid-esophageal xPlane study. Upper images show $90^{\circ}$ view with xPlane cursor positioned on the posterior leaflet (A), the orthogonal plane shows septal and posterior leaflets (B). The model (C) explains orientation of the imaging planes marked with redblue and yellow-green edges. Lower images explain the effect of moving the xPlane cursor to the right towards the anterior leaflet (D-F) Abbreviations: LA, left atrial; LV, left ventricular; RA, right atrial; RV, right ventricular; other see Figure 1
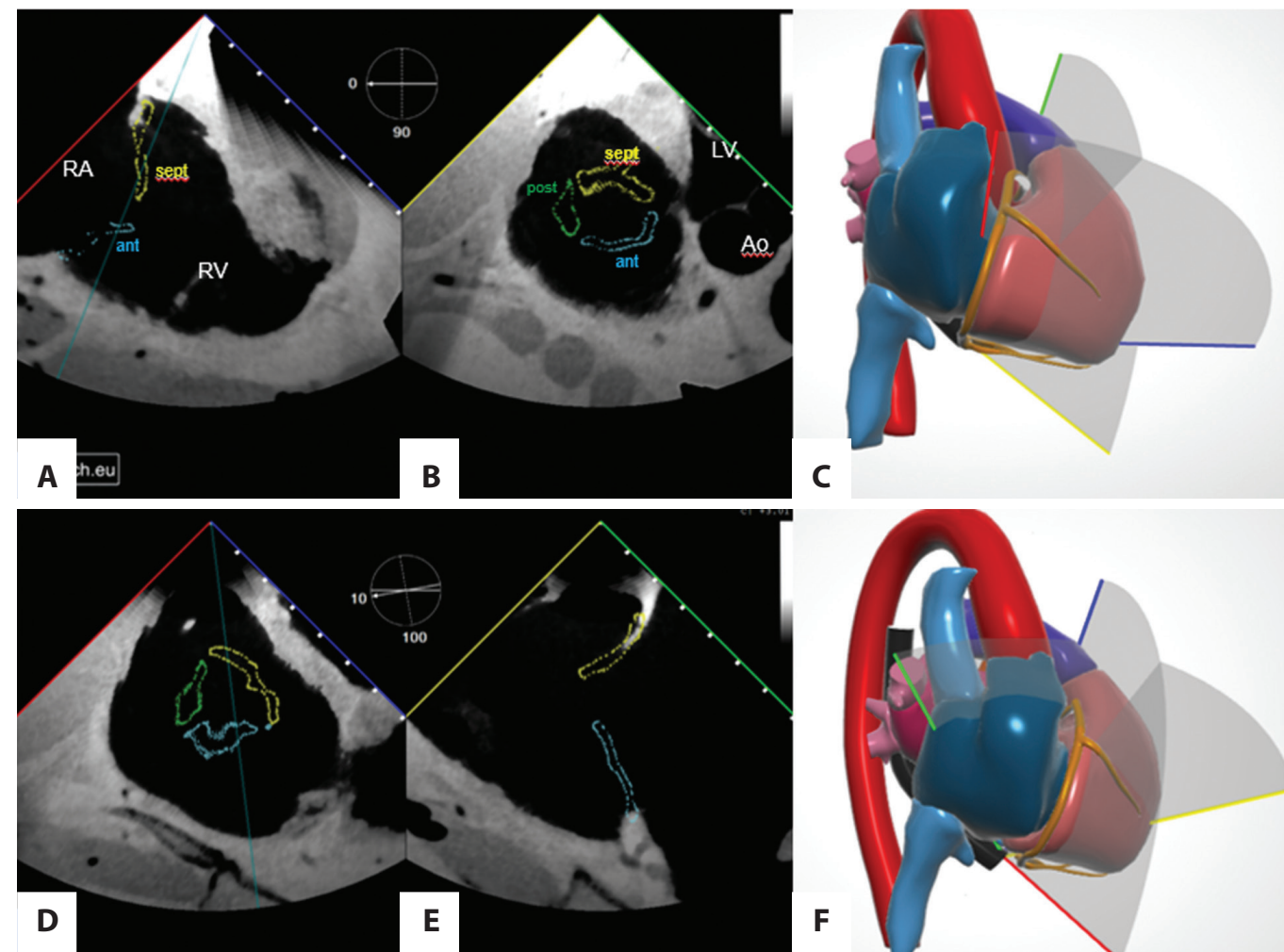

C

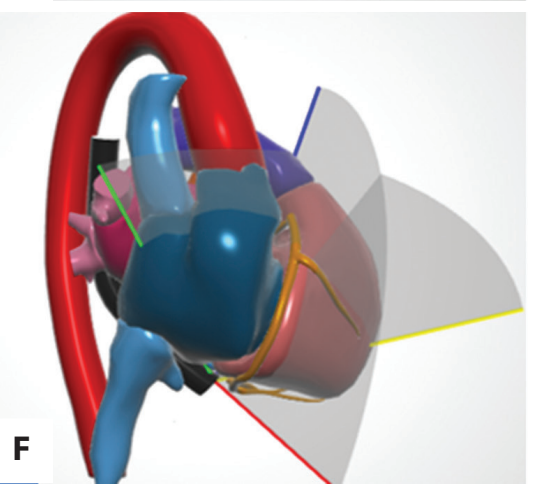

Figure 5. Simulation of the shallow-transgastric xPlane study. The probe is flexed anteriorly and towards the right. Left panel shows the $0^{\circ}$ view with xPlane cursor positioned on the septal and anterior leaflets $(\mathbf{A})$, while the orthogonal plane shows short axis of the valve with all three leaflets (B). The model (C) explains orientation of the imaging planes marked with red-blue and yellow-green edges. The lower panel (D-F) shows the opposite way to obtain similar views. Steering the xPlane cursor on the short image of the valve (D) can orient the long axis plane (E) on the desired leaflets

Abbreviations: see Figures 1 and 4 

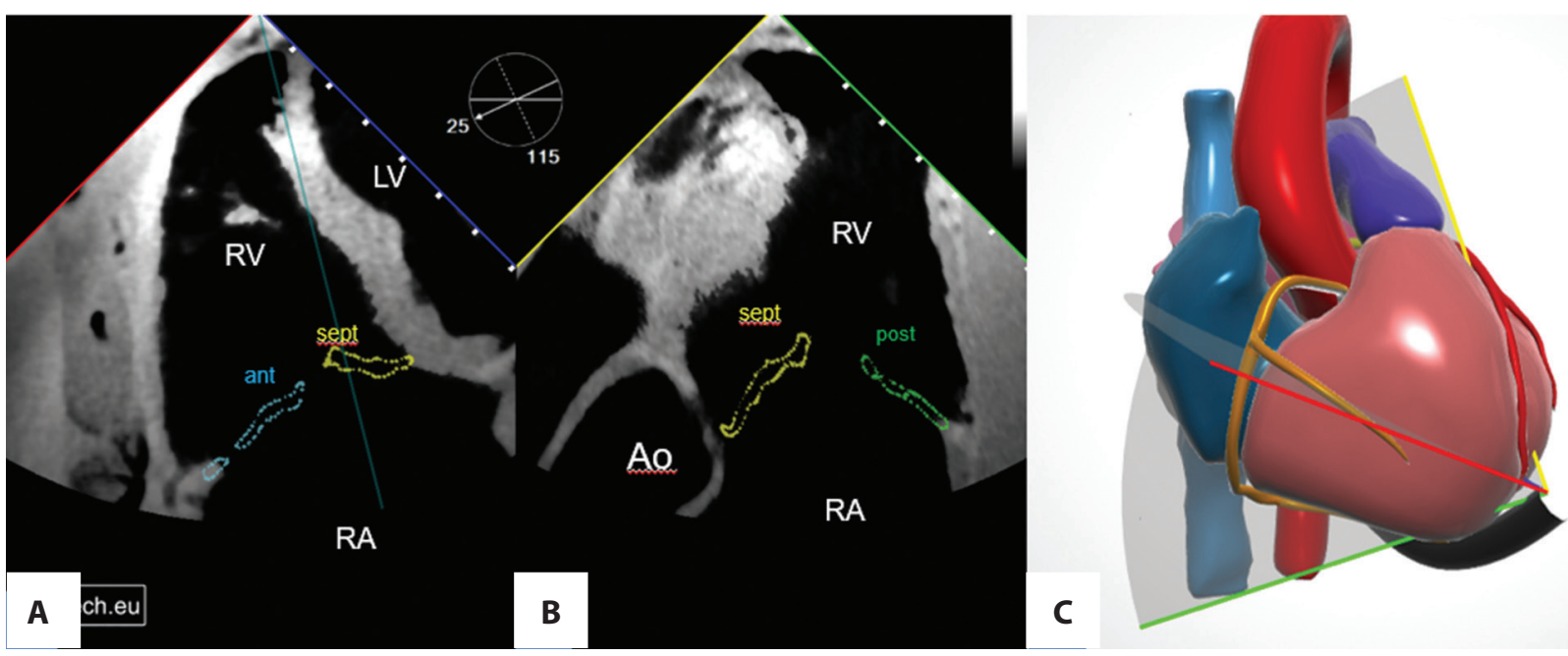

Figure 6. Simulation of the deep-transgastric xPlane study. The probe is maximally anteflexed, showing the long axis of the right ventricle and tricuspid valve (A-B). The model explains orientation of the imaging planes (C)

Abbreviations: see Figures 1 and 4

device is indicated for patients who have severe TR with valve anatomic coaptation gaps of $\leq 1.0 \mathrm{~cm}$, are at high risk for tricuspid valve surgery, do not have severe mitral regurgitation or pulmonary hypertension (systolic pulmonary artery pressure $>60 \mathrm{~mm} \mathrm{Hg}$ ), and are symptomatic despite medical therapy [22]. Contraindications include rheumatic tricuspid valve disease, active endocarditis, and thrombi both intracardiac and located in vena cava or femoral veins. The device is not approved for TR associated with congenital tricuspid valve lesions. The required minimum leaflet insertion ( $6 \mathrm{~mm}$ for smaller and $9 \mathrm{~mm}$ for larger devices) defines the minimum mobile leaflet length as $9 \mathrm{~mm}$ and $12 \mathrm{~mm}$ for NT and XT clips, respectively. As leaflet grasping may potentially cause valvular stenosis, a pre-implant mean pressure gradient $\leq 3 \mathrm{~mm} \mathrm{Hg}$ is recommended (mean pressure gradient of $\geq 5 \mathrm{~mm} \mathrm{Hg}$ should be considered a significant risk factor for creating tricuspid valve stenosis).

According to the manufacturer's instructions, the Edwards PASCAL transcatheter valve repair system is indicated for the percutaneous reconstruction of an insufficient or tricuspid valve through tissue approximation [23]. Echocardiographic contraindications include the inability to complete the screening with TEE and the presence of an intracardiac mass, thrombus, or vegetation. The operator should also consider the following anatomic patient characteristics: non-degenerative tricuspid valve disease, evidence of moderate to severe calcification in the grasping area, severe calcification in the annulus or subvalvular apparatus, presence of significant cleft or perforation in the grasping area, as well as leaflet mobility length $<8 \mathrm{~mm}$.

The optimal clinical echocardiographic criteria for percutaneous tricuspid edge-to-edge valve repair are still evolving. Clinical practice guidelines on the management of valvular heart disease provide no formal indications in the selection of patients for percutaneous edge-to-edge tricuspid valve repair.

Baseline right ventricular (RV) size and function, as well as estimates of systolic pulmonary artery pressure, did not predict clinical outcomes after transcatheter tricuspid valve repair in a substudy of the TriValve Registry [24]. Nevertheless, coaptation gap ( $\leq 7 \mathrm{~mm})$, central or anteroseptal jet location, and tethering height $(\leq 1 \mathrm{~cm})$ determined procedural success, which was associated with improved survival [25]. Percutaneous tricuspid edge-to-edge valve repair is performed mainly in patients with torrential, massive, or severe TR. Patients with moderate-to-severe TR, may also sometimes be considered [26].

In summary: echocardiographic criteria predictive of procedural success include:

- $\quad \leq 7$ (10) mm coaptation gap;

- $\quad$ 7 (8) mm leaflet length;

- $\leq 10 \mathrm{~mm}$ tethering height;

- $\leq 3$ (5) mm Hg mean tricuspid pressure gradient. Echocardiographic exclusion criteria include:

- Technically inadequate examinations;

- Rheumatic/congenital etiology;

- Active endocarditis;

- Intracardiac thrombi;

- Significant leaflet and annular calcifications.

\section{ECHOCARDIOGRAPHIC INTRAOPERATIVE GUIDANCE DURING THE TRICUSPID TRANSCATHETER EDGE-TO-EDGE REPAIR}

\section{Phase 1. Navigating to the Tricuspid Valve}

During the tricuspid transcatheter edge-to-edge repair, the steerable guide catheter (SGC) is introduced into the inferior vena cava to the right atrium (RA) using a modified bi-caval view $\left(90^{\circ}-110^{\circ}\right)$. After losing contact with the in- 
teratrial septum, in the middle of the right atrium, the clip delivery system (CDS) should be gradually pushed out from the SGC. The next step is to flex the CDS by moving the tip of the system proximally to the tricuspid valve annulus. This step of the procedure is guided in multi-plane 2D-TEE or real-time 3D echocardiography (RT3DTEE, zoom acquired from $90^{\circ}-110^{\circ}$ ). These views allow one to follow the tip of the system in the right atrium.

\section{Phase 2. Navigating to the Implantation Zone}

Navigation to the area of implantation depends on the coaptation line which is the target of the procedure: antero-septal and/or postero-septal. The following steps are used for setting the device over the chosen area: the basal view used for the navigation to the coaptation line antero-septal/postero-septal is the Right Ventricle Outflow Tract (RVOT) View $\left(60^{\circ}-100^{\circ}\right)$ obtained from the high $/ \mathrm{mid}$ esophagus with the device placed medially, close to the aortic valve (AV). In the same reference plane, if the coaptation line postero-septal is chosen as the implantation target, the device should be moved laterally to the wall of the RA/RV. The use of bi-plane TEE helps with positional assessment in the medio-lateral axis. The position of the device is easy to assess when RT3DTEE is used. The RT3DTEE Zoom volume dataset is acquired from a mid-esophageal 4 chamber view $\left(0^{\circ}-20^{\circ}\right.$ or $\left.160^{\circ}-180^{\circ}\right)$. The volume should include anatomical landmarks: aortic valve (non-coronary sinus), intra-atrial septum, right atrial appendage, and superior vena cava.

\section{Phase 3. Implantation}

After confirmation of the device position over the desired implantation area, the perpendicularity of the clip to the coaptation line should be assessed. When the device is close to the leaflets, the arms of the clip and coaptation line can be seen using the trans-gastric short-axis view $\left(20^{\circ}-50^{\circ}\right)$. In the in the latter view, the parallax artifact can interfere with the proper perpendicularity adjustment. When perpendicularity is confirmed, the clip should be closed again. In the majority of cases, a similar view can be acquired from RT3DTEE zoom as described above.

Under constant monitoring in several views, the device should be placed in the right ventricle just below the tricuspid leaflets to avoid entrapment in the subvalvular apparatus. This part of the procedure can be monitored using the trans-gastric long-axis view. However, the mid-esophageal 4 chamber view $\left(0^{\circ}-20^{\circ}\right.$ or $\left.160^{\circ}-180^{\circ}\right)$ or bi-plane echocardiography based on the RVOT view (reference plane $60^{\circ}-100^{\circ}$ ) can be also used. The latter is very helpful in cases with difficult anatomy or planned postero-septal implantation.

The perpendicularity to the coaptation line should be confirmed when the device is under the leaflets. This can be assessed using the trans-gastric short-axis view or mid-esophageal RT3DTEE zoom. Implantation in the antero-septal position can be monitored from the mid-esophageal
4 chamber view $\left(0^{\circ}-20^{\circ}\right.$ or alternate $\left.160^{\circ}-180^{\circ}\right)$. During PS position implantation, monitoring from the deep-esophageal 4 chamber view (with coronary sinus in sight, $0^{\circ}-20^{\circ}$ or alternate $160^{\circ}-180^{\circ}$ ) might be used. However, in cases of unfavorable anatomy, the RVOT bi-plane view (as the reference plane) may be helpful to monitor the grasping of the leaflets and the closing of the device.

\section{Phase 4. Confirmation of proper implantation.}

Post implantation evaluation includes a leaflet insertion assessment. The remaining part of a leaflet (distance between the device and annulus) should be measured using the echocardiographic view used for implantation monitoring. Following the proper implantation, using the transgastric short-axis view, the valve should become double-orifice if one clip was implanted, or tri-orifice if 2 clips were used. The reduction in TR is checked by color Doppler in the high/mid esophageal 2D RVOT view, bi-plane view, or using RT3DTEE. The trans-gastric long axis view can also be useful in measuring TR reduction. Finally, using a continuous wave Doppler, a measurement of the mean gradient through the tricuspid valve should be obtained, which should not exceed $5 \mathrm{~mm} \mathrm{Hg}$. In some cases, additional measurement during the transthoracic examination might be helpful to rule out the possibility of tricuspid stenosis.

\section{TRICUSPID REPAIR WITH THE MITRACLIP SYSTEM}

Most of the percutaneous tricuspid repair procedures reported to date have been performed with the MitraClip system. This device has not been approved for the treatment of TR, and therefore, its use is considered off-label and should be limited to investigational purposes or compassionate procedures. Since the system was developed for the treatment of mitral valve dysfunction, its use in the right atrium causes it to lose much of its steerability and requires different handling. There are several techniques described, the following, most often used in our practice is one of the possible options.

Proper TEE imaging with clear visualization of the right atrium, tricuspid valve, and its leaflets, especially at the site of planned clip implantation is the most important element of the procedure. Fluoroscopic guidance requiring an RAO $30^{\circ}$ view played an additional role in most cases. Because of the reimbursement restrictions, all the TR repairs in our practice were combined mitral tricuspid interventions. In this setting, the tricuspid repair starts after the successful completion of the mitral procedure, if the TEE shows a persistent TR jet. The procedural steps are descripted on Figure 7.

The main regurgitation jet should be identified using the short axis trans-gastric (TG-SAX) and 3D view. The tip of the clip should be pointed at the desired position using the basic SGC maneuvers presented in Figure 8. In the majority of cases, the clip is placed between the septal and anterior leaflets or, less frequently, between the 


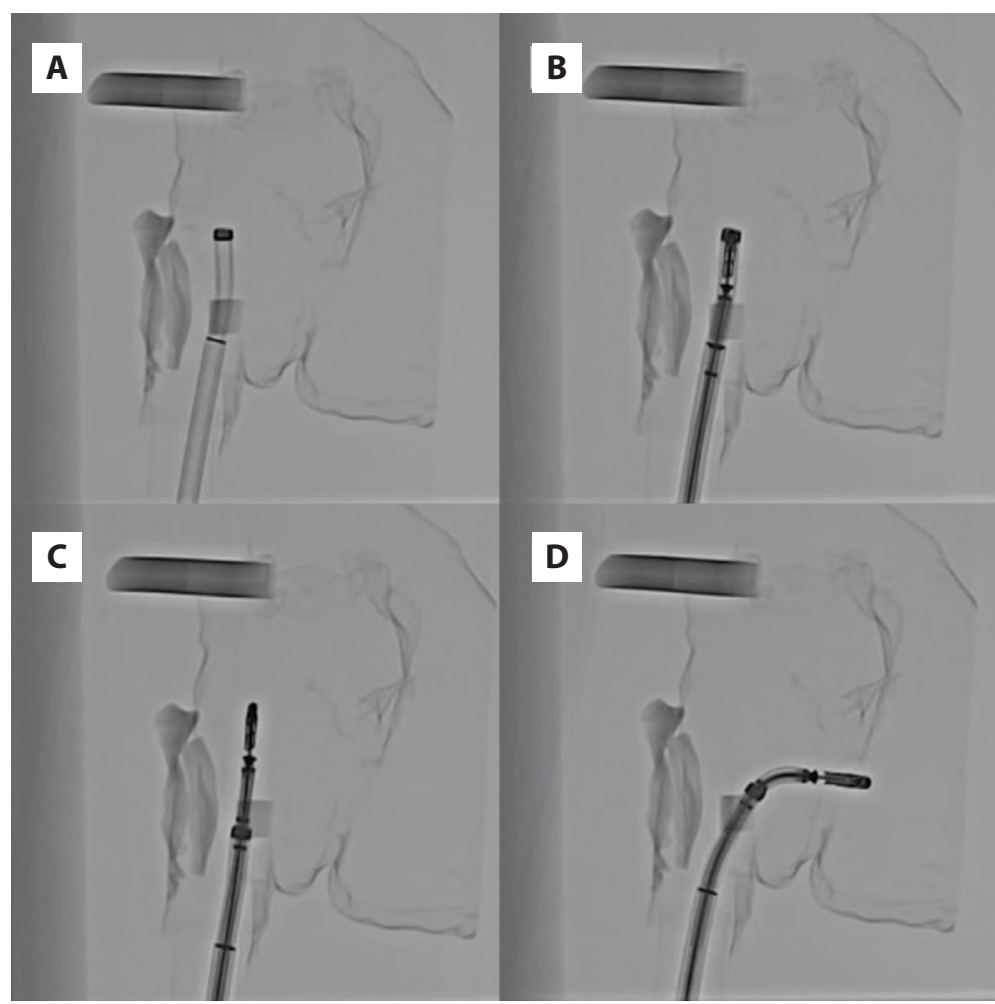

Figure 7. The procedural steps of the MitraClip insertion for tricuspid regurgitation repair (see also Figure 8). After the addition of "minus" on an SGC +/- knob, the straightened system is slowly removed from the intra-atrial septum (bicaval view or right atrium 3D view) (A). CDS is inserted into the SGC in a "mis-key" manner by turning the CDS $90^{\circ}$ counterclockwise from the "bluetoblue" position. CDS is then advanced to the end of the SGC under fluoroscopic guidance (bicaval view or right atrium 3D view) (B). While the end of the clip is kept in the same position, the $\mathrm{SGC}$ is withdrawn to obtain straddling (bicaval view or right atrium $3 \mathrm{D}$ view) (C). SGC is rotated counterclockwise to point the clip at the TV. On CDS A/P knob "P" is added to steer down the tip of the clip to the TV (right atrium 3D view) (D)

Abbreviations: CDS, clip delivery system; SGC, steerable guide catheter
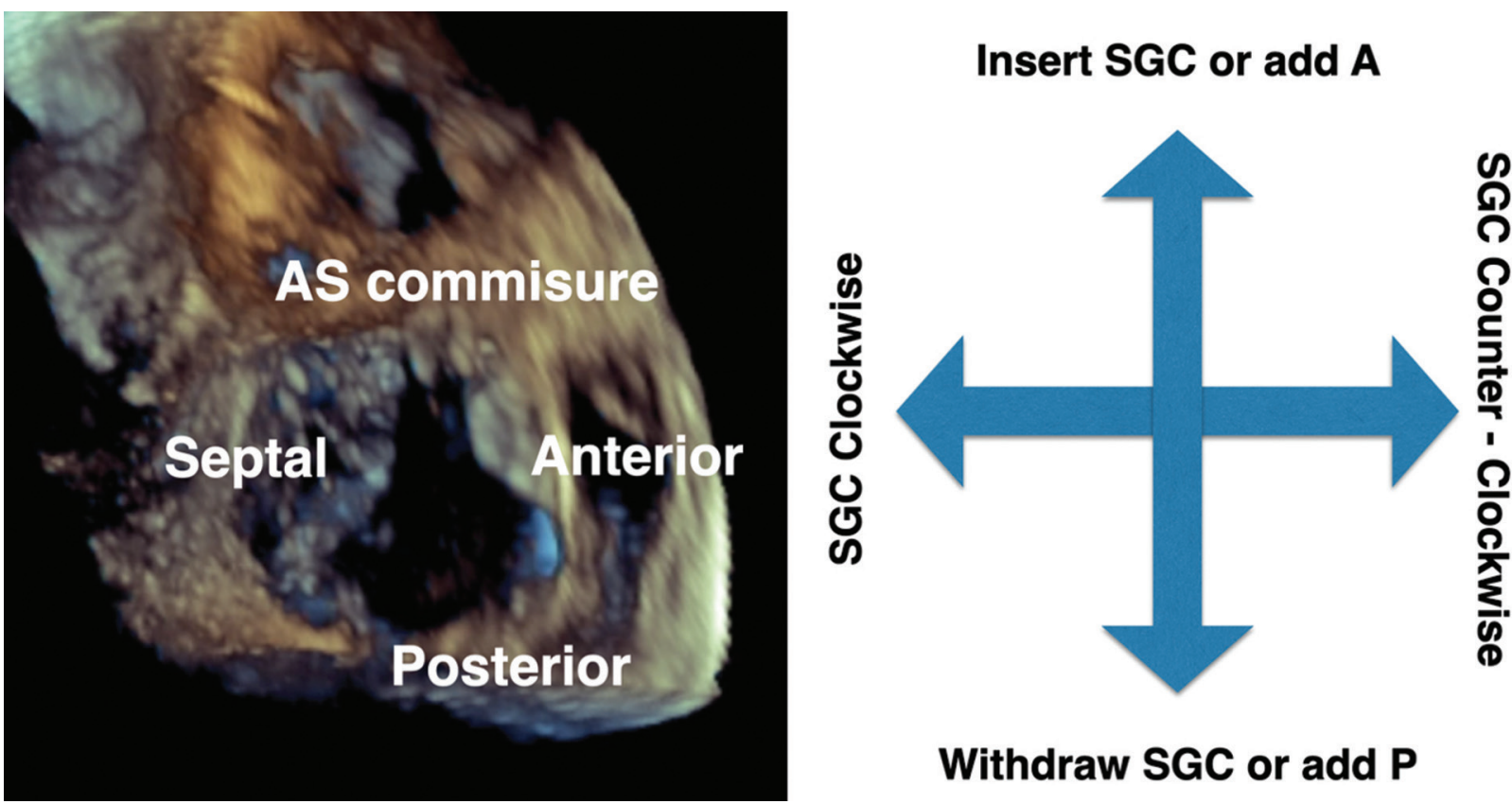

Withdraw SGC or add P

Figure 8. Basic maneuvers for the steerable guide catheter (SGC) orientation in the tricuspid valve 


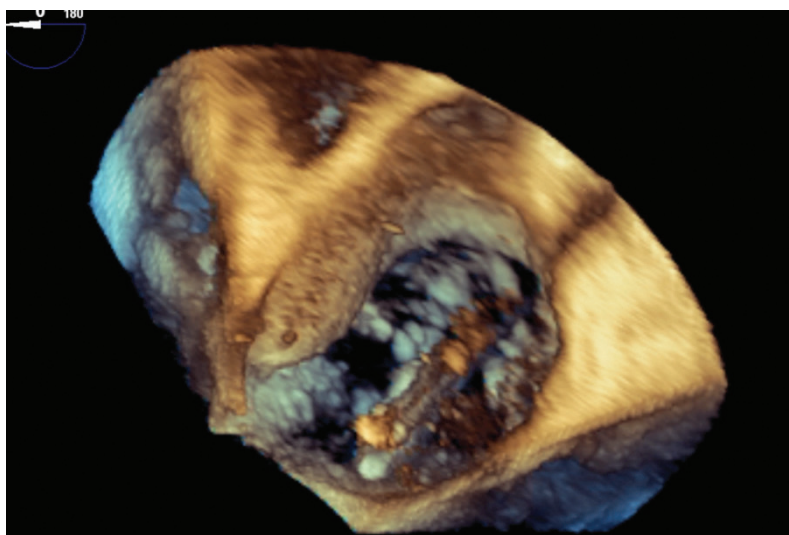

Figure 9. Perpendicularity adjustment in the 3D view

septal and posterior leaflets. Once in position, the clip is opened and the perpendicularity of the arms to the coaptation line is adjusted (TG-SAX, 3D) (Figure 9). After insertion of the system into the right ventricle, grasping of a leaflet can be attempted using the mid-esophageal views (Figure 10). The grasping can be confirmed by direct visualization of the leaflet insertion in the mid-esophageal views, by the reduction of regurgitation jet, and by leaflet immobilization visible in the TG-SAX view (Figure 11). Procedural success is defined as at least one grade reduction in TR severity and lack of a TV mean gradient of more than $3 \mathrm{~mm} \mathrm{Hg}$.

The MitraClip system's off-label use for this indication has several technical limitations which might hamper effective TV repair. In some patients, insufficient space between the inferior vena cava and the TV might result in low positioning of the device with the clip below the TV plane despite full CDS handle retraction. This might prevent successful grasping and result in clip entrapment in the right ventricle with an inability to move it into the right atrium. What is more, the unfavorable orientation of the inferior vena cava and the plane of the valve might cause a phenomenon known as "septal hugging" in which the path of the device is not perpendicular to the TV plane (Figure 12). These issues have mostly been resolved by the redesigned shape and additional steering options of the TriClip, a percutaneous system developed for the treatment of TR.

\section{THE TRICLIP SYSTEM}

The TriClip device is a first-in-class transcatheter edgeto-edge repair system dedicated to a minimally invasive, percutaneous treatment of TR. The TriClip system evolved from the MitraClip NTR platform, which is commonly and successfully used not only for mitral valve repair but also for TR treatment [27]. Both MitraClip and TriClip systems consist of an SGC and a CDS with attached cobalt-chromium clips available in two sizes NT and XT (implant arm length 9 and $12 \mathrm{~mm}$, respectively). When compared to the MitraClip, the SGC of the TriClip has two knobs ( $/ L$ and \pm ) and CDS one knob (F/E) for the multi-directional steering maneuvers and deflection of the system. Modification of the MitraClip system was crucial in overcoming the socalled septal huger phenomenon, defined as the tendency to self-position the delivery catheter towards the septal leaflet but also for precise control of perpendicularity and height adjustments.

The efficacy and safety of the TriClip system were established in the TRILUMINATE trial, which was a prospective, single-arm, feasibility study conducted at 21 sites in Europe and the USA. A total of 85 symptomatic patients with moderate or greater TR underwent a successful procedure (100\% implant success rate) [28]. On average 2.2 TriClips were implanted, with a mean procedure time of 153 minutes. In the majority of cases (77\%), the clips were located in the antero-septal commissure. The primary efficacy endpoint, defined as a reduction of TR

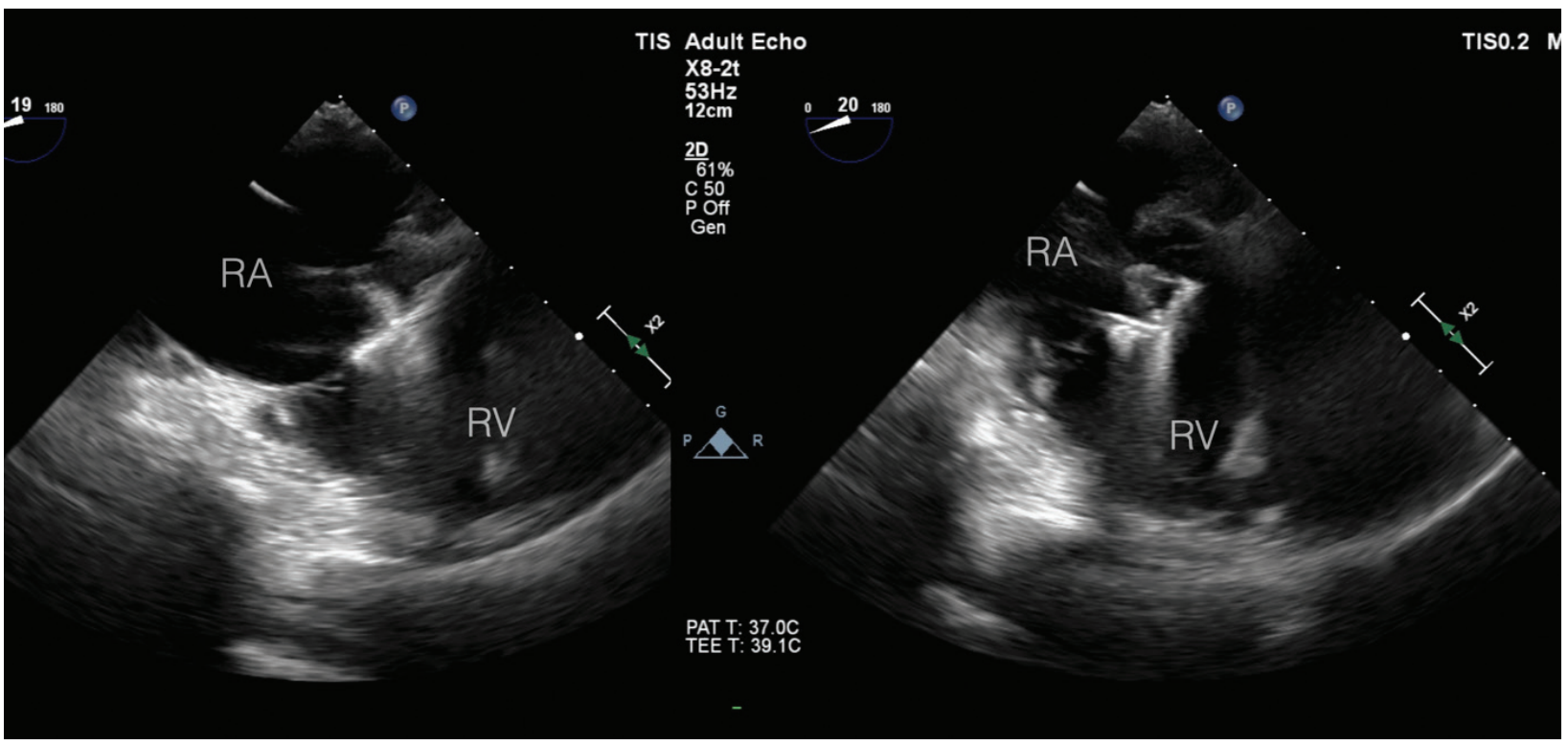

Figure 10. Grasping attempt in the mid esophageal view 


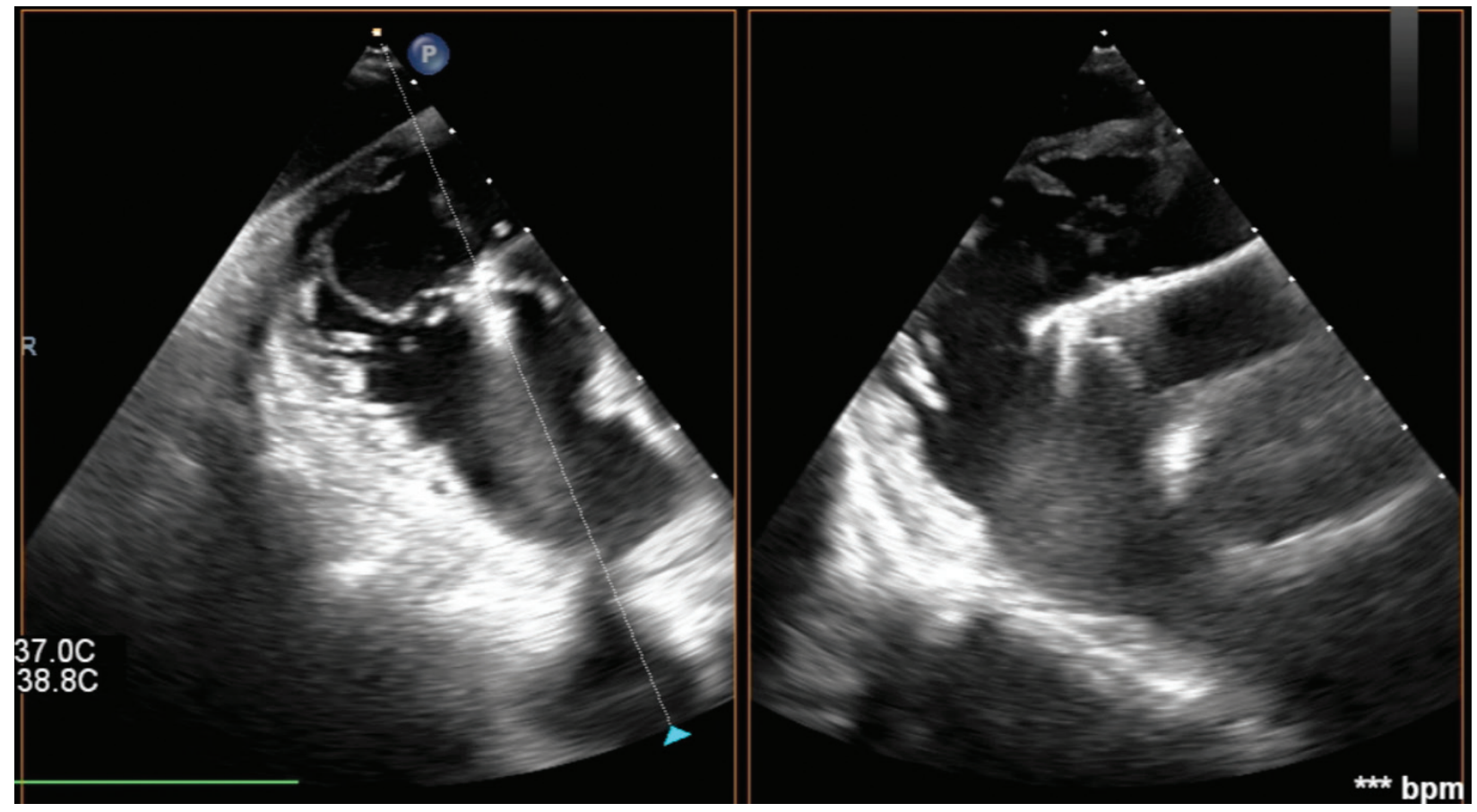

Figure 11. Grasping confirmation in the trans-gastric view

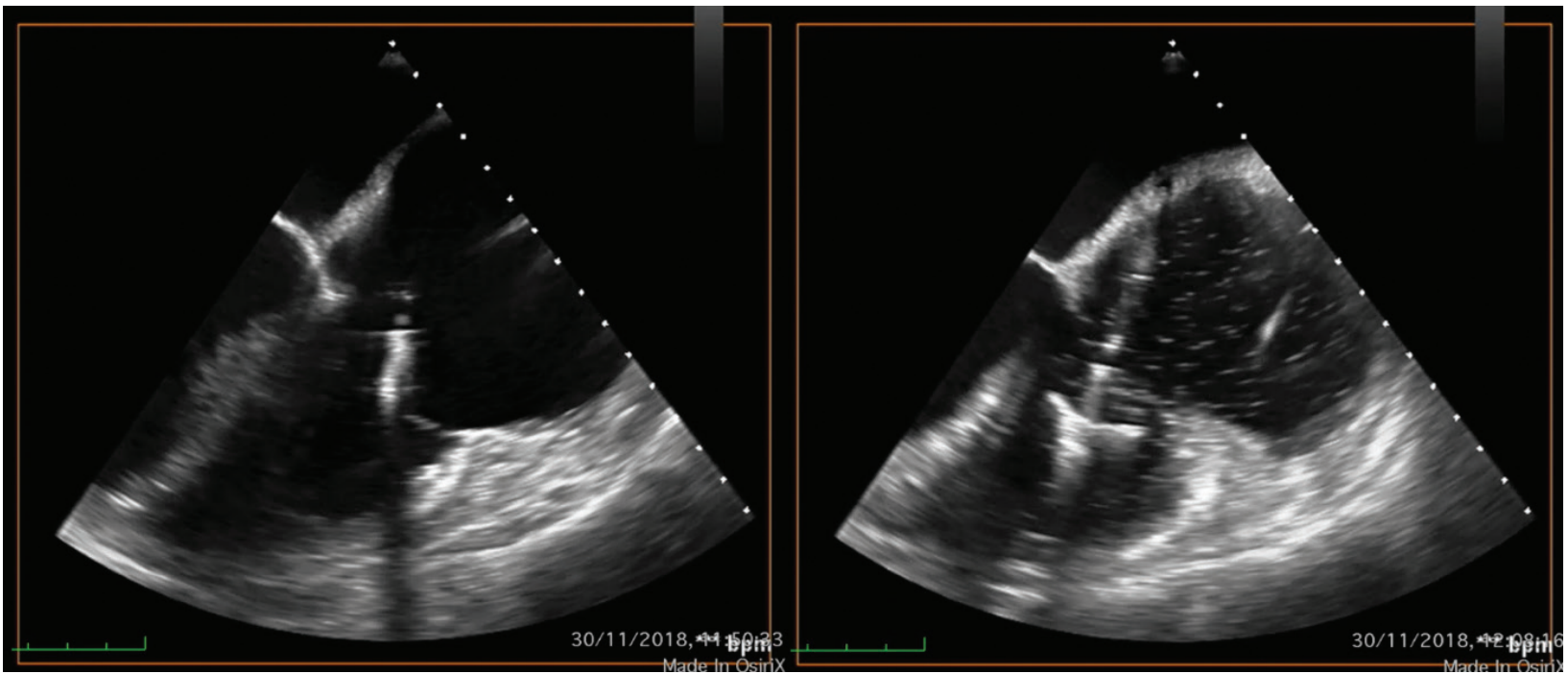

Figure 12. "Septal hugging" — clip path not perpendicular to the plain of the valve

severity by at least one grade at 30 days, was met in $86 \%$ of patients. At 1 year, a reduction in TR was sustained with $71 \%$ of the cases being classified as moderate or less TR, especially when compared with $8 \%$ at baseline $(P<0.0001)$. Echocardiographic assessment performed by the core lab revealed significant positive right heart remodeling in terms of a reduction of the right ventricular end-diastolic diameter $(5.3$ vs. $4.8 \mathrm{~cm} ; P<0.0001)$, a decrease in the right atrial volume (129 vs. $116 \mathrm{ml}$; $P=0.0166)$, and a TAPSE improvement $(1.44$ vs. $1.59 \mathrm{~cm}$; $P=0.0002)$. Patients treated with the TriClip device experienced a significant clinical improvement in NYHA functional class (percentage of patients with NYHA class I/II $31 \%$ vs. $83 \% ; P<0.0001$ ) and exercise capacity (6-minute walk test increased from 272.3 to 303.2 meters; $P=0.0023)$. The edge-to-edge therapy was also associated with substantial quality-of-life improvements and reduction in hospitalizations. The annual hospitalization rate decreased by $40 \%$ when compared to one year before the procedure $(P=0.003)$.

These results were achieved with few safety concerns such as major bleeding observed in 10 patients, 5 cases of single leaflet device attachment, and 4 cases with tricuspid valve mean gradient equal or greater than $5 \mathrm{~mm} \mathrm{Hg}$. The occurrence of major adverse events through 1 year was also low with a total number of 6 events, including 4 cases of cardiovascular death. Safety and effectiveness of the TriClip procedure are currently under investigation in the pivotal 
trial TRILUMINATE (Clinical TRlal to EvaLUate Cardiovascular OutcoMes IN Patients TreATEd with the Tricuspid Valve Repair System), which is a prospective, randomized study comparing the tricuspid valve edge-to-edge repair system (on top of medical therapy) to pharmacotherapy only in patients with severe, symptomatic TR [29].

\section{TRICUSPID REPAIR WITH THE PASCAL SYSTEM}

The PASCAL transcatheter valve repair system (Edwards Lifesciences, Irvine, CA, USA) is a leaflet repair therapy initially designed for the treatment of MR, which shares the concept of edge-to-edge coaptation enhancement [30, 31]. Technical characteristics of both delivery systems and clipping devices appear to make the PASCAL transcatheter valve repair system a useful tool in the treatment of TR. It uses a pair of clasps and paddles designed to plicate valve leaflets and facilitate coaptation. The broad contoured paddles are designed to maximize leaflet coaptation and minimize stress concentration on the native leaflets. An anatomic spacer is used to fill the regurgitant orifice between the native valve leaflets to prevent backflow, further reducing regurgitant flow. The clasps are adjustable, which aids in the successful positioning of the leaflets. The clasps can be operated either simultaneously or independently to facilitate optimized leaflet capture in cases with complex anatomy. The delivery system consists of a 22-F guide sheath, with 3 independent catheters that facilitate maneuvering in 3 different planes and stabilizers that lock catheter handles in place. The PASCAL repair system implant can be elongated within the subvalvular anatomy to substantially decrease the implant profile for an atraumatic repositioning if deemed necessary.

The CLASP study (Edwards PASCAL Transcatheter Mitral Valve Repair System Study) was designed to assess the clinical benefit of PASCAL edge-to-edge mitral intervention. Results at 1-year follow-up in functional mitral regurgitation and degenerative mitral regurgitation were recently published [32]. Based on the convincingly positive results of this study, it was concluded that the PASCAL transcatheter valve repair system demonstrated a low complication rate, high survival rate, and a sustained MR reduction resulting in significant improvements in functional status and quality of life at 1 year.

The results of an observational first-in-human assessment of feasibility and safety of the PASCAL transcatheter valve repair system and its impact on short-term clinical outcomes in patients with severe TR were encouraging [33]. Twenty-eight patients with severe TR were treated with the PASCAL system in a compassionate use intervention at 6 sites. All patients had heart failure due to severe TR and were deemed to be a high surgical risk by local heart teams. The primary outcome was a procedural success, defined as the implantation of at least 1 device with post-procedural TR grade $\leq 2+$, without mortality or conversion to surgery. TR etiology was functional in $92 \%$ of patients, with a mean tricuspid annular diameter of $49.5 \pm 6 \mathrm{~mm}$ and a mean coaptation gap of $6.9 \pm 3 \mathrm{~mm}$. The procedural success was $86 \%$, with $1.4 \pm 0.6$ devices implanted per patient. There were no intraprocedural complications. At 30-day follow-up, mortality was 7.1\%, $88 \%$ of patients were in NYHA functional class I or II, and $85 \%$ had a TR grade of $\leq 2+$. There were 2 single-leaflet device attachments, which were managed conservatively. The six-minute walk distance improved from 240 m (interquartile range: 172 to $337 \mathrm{~m}$ ) to $335 \mathrm{~m}$ (interquartile range: 251 to $385 \mathrm{~m})(P<0.001)$. This first-in-human experience evaluating transcatheter tricuspid repair with the PASCAL system demonstrated a high procedural success, acceptable safety, and significant clinical improvement.

Efficacy and safety of PASCAL therapy in TR patients is further studied in an ongoing CLASP TR Early Feasibility Study. Of the 34 patients enrolled in this study, the mean age was 76 years, 53\% were women, the mean Society of Thoracic Surgeons score was $7.3 \%, 88 \%$ had atrial fibrillation/flutter, 97\% had severe or greater TR, and 79\% had NYHA functional class III/IV symptoms. Twenty-nine patients (85\%) received implants. Tricuspid regurgitation severity reduction of at least 1 grade at 30 days was achieved in $85 \%$ of them, with $52 \%$ with moderate or less TR $(P<0.001)$. The MAE rate was $5.9 \%$ and none of the patients experienced cardiovascular mortality, stroke, myocardial infarction, renal complication, or reintervention. Eightynine percent of patients improved to NYHA functional class I/II $(P<0.001)$, improved their mean 6-min walk distance by $71 \mathrm{~m}(P<0.001)$, and improved the mean Kansas City Cardiomyopathy Questionnaire score by 15 points $(P<0.001)$. Investigators concluded that in this early experience, the repair system performed as intended, with substantial TR reduction, a low adverse events rate, no mortality or reintervention, and significant improvements in functional status, exercise capacity, and quality of life [34].

\section{SUMMARY}

The approach to the management of valvular heart diseases was completely transformed by the advent of transcatheter valvular interventions. The use of transcatheter valve therapies allowed an expansion of indications to patients previously deemed inoperable. The TR was undertreated for a long time, despite its adverse impact on clinical outcomes, including mortality and heart failure, as well as associated poor quality of life. The contemporary TR treatment was redefined by several important discoveries. Since the first procedure of TR edge-to-edge-repair by Nickenig and colleagues, there has been growing evidence from registries (TriValve and others) and more recently from prospective clinical trials (Triluminate) that edge-to-edge repair using the MitraClip or more recently the TriClip provides a safe and effective treatment, with over $80 \%$ of patients having a significant reduction of TR to moderate or less postoperatively, which is sustained above $70 \%$ at one year. It also leads to a reduction of rehospitalizations and improvement 
in symptoms [26]. Importantly, several prognostic factors related to the anatomy of the TV, clinical status (frailty, short life expectancy, severe RV failure, irreversible pulmonary hypertension) were identified. There is also a trend towards improved survival in patients undergoing transcatheter tricuspid repair in comparison with those treated conservatively. These studies have helped identify the patients in whom the therapy is unlikely to produce durable improvements and who should not be treated with a transcatheter repair. The technical feasibility of the procedure will surely evolve as operators gain clinical experience. This will lead to a higher rate of acceptance for the procedure as well as a higher rate of technical success. In addition, multiple new technologies based on transcatheter annuloplasty, implantation of valves in caval veins, as well as dedicated tricuspid bioprosthetic valves mounted on self-expandable stents were designed and are currently undergoing clinical evaluation.

From the Polish perspective, it is important to share our initial experience with heart valve centers experienced with mitral and tricuspid procedures, train operators and echocardiographers to build up the referral network, and follow-up patients within the registry. It seems that at least three groups of patients are likely to be discussed by Heart Teams: secondary TR coexisting with significant MR planned for one step mitral and tricuspid clipping, patients after left-sided valve surgery and significant TR, and patients with isolated TR. Last, but not least, it is important to secure the funding for these procedures which are currently not reimbursed. The current edition of the Valve-for-Life Initiative by the EAPCI and the European Society of Cardiology in Poland should be especially focused on these unmet clinical needs.

In summary, the transcatheter tricuspid edge-to-edge repair seems to be a safe and possibly effective treatment for patients with heart failure and significant TR, leading to the reduction of its severity and clinical improvement in a significant number of patients. This field is rapidly expanding, and Poland joined the group of countries which can offer carefully selected patients access to this innovative and effective therapy.

\section{Article information}

Conflict of interest: AR received proctoring fees and speakers' honoraria from Abbott. PS received proctoring fees from Abbott. AG received proctoring fees and speakers' honoraria from Abbott and Edwards; Co-founder of Medical Simulation Technologies. PŚ received proctoring fees and speakers' honoraria from Abbott. PG received proctoring fees and speakers' honoraria from Abbott and Boston Scientific. AP received proctoring fees and speakers' honoraria from Abbott. JT received proctoring fees from Abbott. JK received proctoring fees and speakers' honoraria from Abbott. AW received speaker's fees from Abbott. WW received speakers' honoraria from Abbott and Edwards Lifesciences. MG received speakers' honoraria Abbott. KZ declares no conflict of interest.

Open access: This article is available in open access under Creative Common Attribution-Non-Commercial-No Derivatives 4.0 International (CC BY-NC-ND 4.0) license, allowing to download articles and share them with others as long as they credit the authors and the publisher, but without permission to change them in any way or use them commercially. For commercial use, please contact the journal office at kardiologiapolska@ptkardio.pl.

How to cite: Rdzanek A, Szymański P, Gackowski A, et al. Percutaneous tricuspid edge-to-edge repair — patient selection, imaging considerations, and the procedural technique. Expert opinion of the Working Group on Echocardiography and Association of Cardiovascular Interventions of the Polish Cardiac Society. Kardiol Pol. 2021; 79(10): 1178-1191, doi: 10.33963/KP.a2021.0125.

\section{REFERENCES}

1. Topilsky Y, Maltais S, Medina Inojosa J, et al. Burden of tricuspid regurgitation in Patients diagnosed in the community setting. JACC Cardiovasc Imaging. 2019; 12(3): 433-442, doi: 10.1016/j.jcmg.2018.06.014, indexed in Pubmed: 30121261.

2. Silbiger JJ. Atrial functional tricuspid regurgitation: an underappreciated cause of secondary tricuspid regurgitation. Echocardiography. 2019; 36(5): 954-957, doi: 10.1111/echo.14327, indexed in Pubmed: 30919501.

3. Shiran A, Sagie A. Tricuspid regurgitation in mitral valve disease incidence, prognostic implications, mechanism, and management. J Am Coll Cardiol. 2009; 53(5): 401-408, doi: 10.1016/j.jacc.2008.09.048, indexed in Pubmed: 19179197.

4. Bannehr M, Edlinger CR, Kahn U, et al. Natural course of tricuspid regurgitation and prognostic implications. Open Heart. 2021; 8(1): e001529, doi: 10.1136/openhrt-2020-001529, indexed in Pubmed: 33563777.

5. Topilsky Y, Nkomo VT, Vatury O, et al. Clinical outcome of isolated tricuspid regurgitation. JACC Cardiovasc Imaging. 2014; 7(12): 1185-1194, doi: 10.1016/j.jcmg.2014.07.018, indexed in Pubmed: 25440592.

6. Wang N, Fulcher J, Abeysuriya N, et al. Tricuspid regurgitation is associated with increased mortality independent of pulmonary pressures and right heart failure: a systematic review and meta-analysis. Eur Heart J. 2019; 40(5): 476-484, doi: 10.1093/eurheartj/ehy641, indexed in Pubmed: 30351406.

7. Bartko $P$, Arfsten $H$, Frey M, et al. Natural history of functional tricuspid regurgitation: implications of quantitative Doppler assessment. JACC:Cardiovascular Imaging. 2019; 12(3):389-397, doi: 10.1016/j.jcmg.2018.11.021, indexed in Pubmed: 30660536.

8. Schueler R, Öztürk C, Sinning JM, et al. Impact of baseline tricuspid regurgitation on long-term clinical outcomes and survival after interventional edge-to-edge repair for mitral regurgitation. Clin Res Cardiol. 2017; 106(5): 350-358, doi: 10.1007/s00392-016-1062-1, indexed in Pubmed: 27999930.

9. Geyer M, Keller K, Bachmann K, et al. Concomitant tricuspid regurgitation severity and its secondary reduction determine long-term prognosis after transcatheter mitral valve edge-to-edge repair. Clin Res Cardiol. 2021; 110(5): 676-688, doi: 10.1007/s00392-020-01798-4, indexed in Pubmed: 33433670.

10. Wengenmayer T,Zehender M, Bothe W, et al. First transfemoral percutaneous edge-to-edge repair of the tricuspid valve using the MitraClip system. Eurolntervention. 2016; 11(13): 1541-1544, doi: 10.4244/EIJV11I13A296, indexed in Pubmed: 27107316.

11. Nickenig G, Kowalski M, Hausleiter J, et al. Transcatheter treatment of severe tricuspid regurgitation with the edge-to-edge MitraClip technique. Circulation. 2017; 135(19): 1802-1814, doi: 10.1161/CIRCULATIONAHA.116.024848, indexed in Pubmed: 28336788.

12. Orban M, Rommel KP, Ho EC, et al. Transcatheter edge-to-edge tricuspid repair for severe tricuspid regurgitation reduces hospitalizations for heart failure. JACC Heart Fail. 2020; 8(4): 265-276, doi: 10.1016/j. jchf.2019.12.006, indexed in Pubmed: 32241534.

13. Cai S, Bowers N, Dhoot A, et al. Natural history of severe tricuspid regurgitation: outcomes after transcatheter tricuspid valve intervention compared to medical therapy. Int J Cardiol. 2020; 320: 49-54, doi: 10.1016/j. ijcard.2020.07.018, indexed in Pubmed: 32682962.

14. Vahanian A, Beyersdorf F, Praz F, et al. ESC/EACTS Scientific Document Group. 2021 ESC/EACTS Guidelines for the management of valvular heart disease. Eur Heart J. 2021 [Epub ahead of print]: 30660536, doi: 10.1093/eurheartj/ehab395, indexed in Pubmed: 34453165.

15. Otto CM, Nishimura RA, Bonow RO, et al. 2020 ACC/AHA guideline for the management of patients with valvular heart disease: executive sum- 
mary: a report of the American College of Cardiology/American Heart Association Joint Committee on Clinical Practice Guidelines. Circulation. 2021; 143(5): e35-e71, doi: 10.1161/CIR.0000000000000932, indexed in Pubmed: 33332149.

16. Mehr M, Karam N, Taramasso M, et al. Combined tricuspid and mitral versus isolated mitral valve repair for severe MR and TR: an analysis from the TriValve and TRAMI registries. JACC: Cardiovascular Interventions. 2020; 13(5): 543-550, doi: 10.1016/j.jcin.2019.10.023, indexed in Pubmed: 31954679.

17. Taramasso M, Hahn RT, Alessandrini H, et al. The international multicenter TriValve registry: which patients are undergoing transcatheter tricuspid repair? JACC Cardiovasc Interv. 2017; 10(19): 1982-1990, doi: 10.1016/j. jcin.2017.08.011, indexed in Pubmed: 28982563.

18. McCarthy PM, Bhudia SK, Rajeswaran J, et al. Tricuspid valve repair: durability and risk factors for failure. J Thorac Cardiovasc Surg. 2004; 127(3): 674-685, doi: 10.1016/j.jtcvs.2003.11.019, indexed in Pubmed: 15001895.

19. Hahn RT, Zamorano JL. The need for a new tricuspid regurgitation grading scheme. Eur Heart J Cardiovasc Imaging. 2017; 18(12): 1342-1343, doi: 10.1093/ehjci/jex139, indexed in Pubmed: 28977455.

20. Otto CM, Nishimura R, Bonow R, et al. 2020 ACC/AHA guideline for the management of patients with valvular heart disease: a report of the American College of Cardiology/American Heart Association Joint Committee on Cinical Practice Guidelines. Circulation. 2021; 143(5): e72-e227, doi: 10.1161/cir.0000000000000923, indexed in Pubmed: 33332150.

21. Hahn RT. A review of imaging options relevant to treating functional tricuspid regurgitation with transcatheter techniques. Cardiac Interventions Today. 2017; 11(4): 40-47.

22. Instructions for Use TriclipTM System. https://vascular.eifu.abbott/en/detail-screen.html (May 6, 2021).

23. Instructions for use Edwards PASCAL Transcatheter Valve Repair System https://eifu.edwards.com/eifu/pages/viewers/pdf?projectKey=5e7e4f5d87b3940001e289be\&itemKey=605bd73ab4147f0001900c76 (May 6, 2021).

24. Karam N, Mehr M, Taramasso M, et al. Value of echocardiographic right ventricular and pulmonary pressure assessment in predicting transcatheter tricuspid repair outcome. JACC Cardiovasc Interv. 2020; 13(10): 1251 1261, doi: 10.1016/j.jcin.2020.02.028, indexed in Pubmed: 32360260.

25. Besler C, Orban M, Rommel KP, et al. Predictors of procedural and clinical outcomes in patients with symptomatic tricuspid regurgitation undergoing transcatheter edge-to-edge repair. JACC Cardiovasc Interv. 2018; 11(12): 1119-1128, doi: 10.1016/j.jcin.2018.05.002, indexed in Pubmed: 29929631.
26. Mehr M, Taramasso M, Besler C, et al. 1-Year outcomes after edge-to-edge valve repair for symptomatic tricuspid regurgitation: results from the TriValve registry. JACC Cardiovasc Interv. 2019; 12(15): 1451-1461, doi: 10.1016/j.jcin.2019.04.019, indexed in Pubmed: 31395215.

27. Taramasso M, Alessandrini $\mathrm{H}$, Latib A, et al. Outcomes after current transcatheter tricuspid valve intervention: mid-term results from the international TriValve registry. JACC Cardiovasc Interv. 2019; 12(2): 155-165, doi: 10.1016/j.jcin.2018.10.022, indexed in Pubmed: 30594510.

28. Nickenig G, Weber M, Lurz $P$, et al. Transcatheter edge-to-edge repair for reduction of tricuspid regurgitation: 6-month outcomes of the TRILUMINATE single-arm study. Lancet. 2019; 394(10213): 2002-2011, doi: 10.1016/s0140-6736(19)32600-5, indexed in Pubmed: 31708188.

29. Lurz P, Stephan von Bardeleben R, Weber M, et al. TRILUMINATE Investigators. Transcatheter edge-to-edge repair for treatment of tricuspid regurgitation. J Am Coll Cardiol. 2021; 77(3): 229-239, doi: 10.1016/j. jacc.2020.11.038, indexed in Pubmed: 33478646.

30. Praz F, Spargias K, Chrissoheris M, et al. Compassionate use of the PASCAL transcatheter mitral valve repair system for patients with severe mitral regurgitation: a multicentre, prospective, observational, first-in-man study. Lancet. 2017;390(10096): 773-780, doi: 10.1016/S0140-6736(17)31600-8, indexed in Pubmed: 28831993.

31. Lim DS, Kar S, Spargias K, et al. Transcatheter valve repair for patients with mitral regurgitation: 30-day results of the CLASP study. JACC CardiovasC Interv. 2019; 12(14): 1369-1378, doi: 10.1016/j.jcin.2019.04.034, indexed in Pubmed: 31255562.

32. Webb JG, Hensey M, Szerlip M, et al. 1-year outcomes for transcatheter repair in patients with mitral regurgitation from the CLASP study. JACC Cardiovasc Interv. 2020; 13(20): 2344-2357, doi: 10.1016/j.jcin.2020.06.019, indexed in Pubmed: 33092709.

33. Fam NP, Braun D, von Bardeleben RS, et al. Compassionate use of the PASCAL transcatheter valve repair system for severe tricuspid regurgitation: a multicenter, observational, first-in-human experience. JACC Cardiovasc Interv. 2019; 12(24): 2488-2495, doi: 10.1016/j.jcin.2019.09.046, indexed in Pubmed: 31857018.

34. Kodali S, Hahn RT, Eleid MF, et al. CLASP TR EFS Investigators. Feasibility study of the transcatheter valve repair system for severe tricuspid regurgitation. J Am Coll Cardiol. 2021; 77(4): 345-356, doi: 10.1016/j. jacc.2020.11.047, indexed in Pubmed: 33509390. 